Pacific Journal of Mathematics

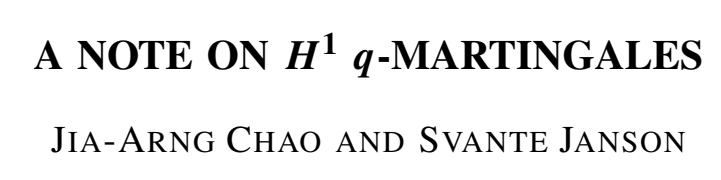




\title{
A NOTE ON $H^{1} q$-MARTINGALES
}

\author{
J.-A. Chao and Svante Janson
}

\begin{abstract}
Characterizations of $H^{1} \quad q$-martingales via conjugate transforms are studied. Applications to lacunary Fourier series and to local field analysis are given.
\end{abstract}

1. Introduction. Characterizations of $H^{1}$ space over a local field and $H^{1}$ regular martingales by singular integral transforms have been studied in a series of papers ([4], [1], [5], [7], and [2]). That is to say,

$$
H^{1}=\left\{f: f, T_{j} f \in L^{1}, j=1,2, \cdots, m\right\}
$$

where $H^{1}$ is the space of regular functions or martingales with integrable maximal functions and $T_{j}, j=1,2, \cdots, m$, are some sort of nice singular integral transforms. In [4], [1] and [5], multiplier transforms arised from multiplicative characters on local fields are used. In [7], singular integral transforms with matrix operators acting on differences of regular martingales are considered. The dyadic case has been excluded until recently. Two methods of handling the dyadic case are given in [2]. First by noting that the maximal function of a dyadic martingale is equivalent in $L^{1}$-norm to the maximal function of its "associated 4-martingale", the space of $H^{1}$ dyadic martingales is characterized by $4 \times 4$ matrix transforms. Then $H^{1}$ space over the dyadic number field is studied via the multiplier transform associated to a multiplicative character of ramification degree 2 .

In this note, we shall extend the above concept of higher ramification degree to $q$-martingales and obtain characterizations for the space $H^{1}$. We also show that the conditions are necessary. These results provide an answer to an open problem posed by Gundy and Varopoulos in [6]. Applications to homogeneous and nonhomogeneous multipliers on local fields are also given.

2. Conjugate characterizations. Let $q$ be an integer larger than 1. Let $\left\{\mathscr{F}_{n}\right\}_{n \geq 0}$ be an increasing sequence of $\sigma$-fields which are generated by atoms in a probability space such that each atom in $\mathscr{F}_{n}$ contains exactly $q$ atoms in $\mathscr{F}_{n+1}$ of equal measure. One example having such a structure is the group $Z_{q}^{\infty}$. Another is the ring of integers of a local field whose residue class field has $q$ elements. A martingale $f=\left\{f_{n}\right\}$ relative to $\left\{\mathscr{F}_{n}\right\}$ is called a $q$-adic martingale or, simply, a q-martingale. 
Let $V_{q}=\left\{\left(x_{i}\right) \in \boldsymbol{C}^{q}: \Sigma_{2} x_{i}=0\right\}$. For a $q$-martingale $f$, the $q$ values of the martingale difference $d_{n}=f_{n}-f_{n-1}$ on $q$ atoms of $\mathscr{F}_{n}$ in a fixed atom of $\mathscr{F}_{n-1}$ is called a local (first) difference of $f$. These local differences can be regarded as vectors in $V_{q}$. Similarly, we call the $q^{k}$ values of $f_{n}-f_{n-k}(n \geqq k \geqq 1)$ on $q^{k}$ atoms of $\mathscr{F}_{n}$ in a fixed atom of $\mathscr{F}_{n-k}$, a local $k$ th difference of $f$.

Associated to each $q$-martingale $f=\left\{f_{n}\right\}_{n \geq 0}$ relative to $\left\{\mathscr{F}_{n}\right\}_{n \geqq 0}$, $F=\left\{f_{n k}\right\}_{n \geq 0}$ is a $q^{k}$-martingale relative to $\left\{\mathscr{F}_{n k}\right\}_{n \geq 0}$. On the other hand, given a $q^{k}$-martingale, we can construct a $q$-martingale in a unique way by filling in the intermediate levels. The local $k$ th differences of a $q$-martingale are local differences of its associated $q^{k}$-martingale. The $H^{1}$ norm of a martingale is taken to be the $L^{1}$-norm of its maximal function. We note that the $H^{1}$ norms of a $q$-martingale and of its associated $q^{k}$-martingale are equivalent. (See e.g., [2] or [6].)

One type of transforms on $q$-martingales was introduced in [7] as follows. Given a $q$-martingale $f$, applying $A$ to each local difference of $f$ on fixed atoms, we obtain another set of local differences which constitute the difference sequence of a $q$-martingale $A f$, say. The transform $f \rightarrow A f$ is a sort of singular integral transform on martingales which is bounded on $H^{p}(0<p<\infty)$ and BMO. (See [7] and [3].) Moreover it is shown in [7] that if $A$ has no real eigenvector in $V_{q}$, then $H^{1}=\left\{f \in L^{1}: A f \in L^{1}\right\}$.

Note that first of all the characterization fails to hold when $q=2$ since $V_{2}$ is a one-dimensional space and so the effect of such a transform $A$ is just multiplying by a constant. Secondly, over local fields, such transforms include only those singular integrals whose kernels are ramified of degree one. We shall now study two related generalizations of these transforms to characterize $H^{1} q$ martingales, both are introduced in [2] for the dyadic case.

The first method is to utilize the one-to-one correspondence between $q$-martingales and their associated $q^{k}$-martingales. Let $A$ be a linear operator on $V_{q^{k}}$. For a $q$-martingale $f$, let $F$ be its associated $q^{k}$-martingale. Apply $A$ to local differences of $F$ to obtain local differences of a $q^{k}$-martingale $A F$ and then construct from it the $q$-martingale $A f$. The result in [7] together with the equivalence of the corresponding $H^{1}$ norms give:

THEOREM 1. Let $A_{j}, j=1,2, \cdots, m$, be linear operators on $V_{q^{k}}$ with $k \geqq 1$. Then $H^{1}=\left\{f: f, A_{j} f \in L^{1}, j=1,2, \cdots, m\right\}$ if any only if $A_{j}, j=1,2, \cdots, m$, do not have a common real eigenvector in $V_{q^{k}}$.

However, this method does not apply to transforms which correspond to multipliers on local fields that are ramified of degree 
$k \geqq 2$. For simplicity, we treat here only the case $k=2$ in detail.

Given a linear operator $A$ on $V_{q}^{q}$, we define the transform $T=$ $T_{A}$ relative to $A$ as follows. Let $f=\left\{f_{n}\right\}$ be a $q$-martingale with martingale difference sequence $\left\{d_{n}\right\}$. On each atom in $\mathscr{F}_{n-2}$, the $q^{2}$ values of $d_{n}$ is a vector in $V_{q}^{q}$. Let $A d_{n}$ denote the image of $d_{n}$ under $A$ on the same atom. Those values of $A d_{n}$ on various atoms form the martingale difference sequence of a $q$-martingale $T f=T_{A} f$. Multipliers of ramification degree 2 on a local field are such transforms. One detailed example is given in [2]. A local second difference of $T f,(T f)_{n}-(T f)_{n-2}=A d_{n}+A d_{n-1}$, on an atom in $\mathscr{F}_{n-2}$, dependes on both $d_{n}$ and $d_{n-1}$. It has values in two levels, i.e., $A d_{n}$ and $A d_{n-1}$, mixed together. This is why the first method does not apply here. Note that if we assume $d_{n}=0$ for all odd $n$, then we have $T f=A f$ where $A f$ is obtained by the first method. Thus one idea for further generalizations is to apply the previous argument to the $q^{2}$-martingales, $\Sigma d_{2 n}$ and $\Sigma A d_{2 n}$, which only involve the martingale difference of even levels. Do the same for $\Sigma d_{2 n-1}$ and $\Sigma A d_{2 n-1}$. We could then characterize $H^{1} q$-martingale $f$ by the $L^{1}$-boundedness of $\Sigma d_{2 n}, \Sigma A d_{2 n}, \Sigma d_{2 n-1}$ and $\Sigma A d_{2 n-1}$.

Moreover, consider such a linear operator on $V_{2}^{2}$ given by the matrix

$$
A=\frac{i}{2}\left[\begin{array}{rrrr}
0 & 0 & 1 & -1 \\
0 & 0 & -1 & 1 \\
-1 & 1 & 0 & 0 \\
1 & -1 & 0 & 0
\end{array}\right]
$$

which corresponds to the multiplier transform $T$ studied in $[2 ; \S 3]$. We observe that $\left[\begin{array}{r}1 \\ 1 \\ -1 \\ -1\end{array}\right]$ is a real eigenvector of $A$ in $V_{4}$. The argument in [7] does not apply to $A$ for 4-martingales. However $A$ has no eigenvector in $\boldsymbol{R}^{4} \cap V_{2}^{2}$. When restricted on $V_{2}^{2}$, the submartingale property involved is still valid as seen in [2]. This suggests that the earlier submartingale results could be sharpened for application to such transforms. The above ideas lead to the following generalization.

For a $q$-martingale $f$, let $E f=\Sigma d_{2 n}$ and $G f=\Sigma d_{2 n-1}$. Note that $E f$ and $G f$ are $q^{2}$-martingales with local differences in $V_{q}^{q}$.

TheOREM 2. Suppose $A_{j}: V_{q}^{q} \rightarrow V_{q}^{q}, j=1,2, \cdots, m$, do not have a common real eigenvector in $V_{q}^{q}$ and $T_{j}=T_{A_{j}}$. Then $E f, T_{j} E f$, Gf and $T_{j} G f, j=1,2, \cdots, m$, are $L^{1}$-bounded if and only if $f \in H^{1}$. 
The necessity of the condition in Theorem 2 is obvious. The proof of the sufficiency will be given in $\S 3$. Another version of the theorem is the following.

COROLlaRY 1. If the linear operator $A$ on $V_{q}^{q}$ has no real eigenvector in $V_{q}^{q}$ and $T=T_{A}$. Then $H^{1}=\left\{f: f, T f, E f, T E f \in L^{1}\right\}$.

These results can be generalized to $k>2$. In that case we have operators $A_{j}$ on $V_{q}^{q^{k-1}} \subset V_{q^{k}}$. The analogue of Theorem 2 holds with $k$ operators in the place of $E$ and $G$, and hence there are $(m+1) k$ transforms (including possibly the identity) involved.

Theorem 2 fails if $A_{j}, j=1,2, \cdots, m$, have a common eigenvector in $\boldsymbol{R}^{q^{2}} \cap V_{q}^{q}$. It is shown in [7] how the eigenvector is used to construct a $q^{2}$-martingale $F$ in $L^{1}$ but not in $H^{1}$ such that $A_{j} F=$ $\lambda_{j} F, \lambda_{j} \in C, j=1,2, \cdots, m$. Let $f$ be the $q$-martingale corresponding to $F$. Then $A_{j} f=\lambda_{j} f, j=1,2, \cdots, m$. The local differences of $F$ are by construction all proportional to the eigenvector and thus are elements in $V_{q}^{q}$. This implies that $E f=f$ and $G f=0$. Also, as noted before, $T_{j} E f=A_{j} E f=A_{j} f=\lambda_{j} f, j=1,2, \cdots, m$. Therefore $E f, T_{j} E f=\lambda_{j} f, G f=0$ and $T_{j} G f=0, j=1,2, \cdots, m$, all belong to $L^{1}$, but $f \notin H^{1}$.

3. Basic lemma. We present here the basic lemma of which Theorem 2 is a consequence.

Lemma. Let $W$ be a closed cone $e^{1}$ consisting of elements of the form $x=\left(x^{1}, \cdots, x^{m}\right)$ where $x^{j}=\left(x_{1}^{j}, \cdots, x_{q}^{j}\right) \in V_{q}$ such that if $x^{j}=$ $\eta_{j}\left(\lambda_{1}, \cdots, \lambda_{q}\right)$ for some $\eta_{j} \in \boldsymbol{C}, j=1,2, \cdots, m$, and $\lambda_{i} \in \boldsymbol{R}, i=1,2, \cdots, q$, then $x=(0,0, \cdots, 0)$. Then there is a positive $p<1$ such that

$$
|a|^{p} \leqq \frac{1}{q} \sum_{i=1}^{q}\left|\left(a^{j}+x_{i}^{j}\right)_{j=1}^{m}\right|^{p}
$$

for $a=\left(a^{j}\right)_{j} \in C^{m}$ and $x=\left(x^{j}\right)_{j} \in W$.

Proof. This follows from essentially the same argument used in [7] which we outline below.

Let $\alpha$ be the maximum of $\sum_{i}\left(\operatorname{Re} \sum_{j} \bar{a}^{j} x_{i}^{j}\right)^{2}$ on the compact set

$$
K_{1}=\left\{(a, x) \in C^{m} \times W:|a|=1 \text { and }\|x\|=\left(\sum_{i, j}\left|x_{i}^{j}\right|^{2}\right)^{1 / 2}=1\right\} .
$$

Schwartz's inequality gives $\alpha \leqq 1$. Equality would imply $\sum_{j} \bar{a}^{j} x_{i}^{j} \in \boldsymbol{R}$ and $a^{j} x_{i}^{k}=\lambda a^{k} x_{i}^{j}$ for some $(a, x) \in K_{1}$ and $\lambda \in R$. Thus we would have $x_{i}^{k}=\sum_{j} \bar{a}^{j} a^{j} x_{i}^{k}=\lambda a^{k} \sum_{j} \bar{a}^{j} x_{i}^{j}$ which contradicts the hypothesis. Hence

\footnotetext{
${ }^{1}$ By a cone $W$, we mean $x \in W$ and $t \geqq 0$ imply $t x \in W$.
} 
$\alpha<1$.

From the series expansion of

$$
\begin{aligned}
\left|\left(a^{j}+x_{i}^{j}\right)_{1}^{m}\right|^{p} & =\left(\sum_{j}\left|a^{j}+x_{i}^{j}\right|^{2}\right)^{p / 2} \\
& =|a|^{p}\left\{1+\frac{2 \operatorname{Re} \sum_{j} \bar{a}^{j} x_{i}^{j}}{|a|^{2}}+\frac{\sum_{j}\left|x_{i}^{j}\right|^{2}}{|a|^{2}}\right\}^{p / 2},
\end{aligned}
$$

we obtain (1) for $\alpha \leqq p \leqq 1$ and $\|x\|<\varepsilon|a|$ for some $\varepsilon>0$.

We now let $\beta$ be the maximum of $|a|$ on the compact set

$$
K_{2}=\left\{(a, x) \in C^{m} \times W: \frac{1}{q} \sum_{i}\left|\left(a^{j}+x_{i}^{j}\right)_{1}^{m}\right|=1 \text { and }\|x\| \geqq \varepsilon|a|\right\} .
$$

From 'Minkowski's inequality, we have $\beta \leqq 1$. Equality holds only if $a^{j}+x_{i}^{j}=\lambda_{i} a^{j}$ with $\lambda_{i} \geqq 0$. Thus we would have $x_{i}^{j}=\left(\lambda_{i}-1\right) a^{j}$ which contradicts again the hypothesis.

Therefore, if $\|x\| \geqq \varepsilon|a|$, then $|a| \leqq(\beta / q) \sum_{i}\left|\left(a^{j}+x_{1}^{j}\right)_{1}^{m}\right|$ with $\beta<1$ from which (1) follows if $(\beta / q)^{p} \leqq 1 / q$. This proves the lemma.

We note that the lemma includes the earlier versions of subregularity (or submartingale property) in [1], [4], [5] and [7] as special cases.

We now complete the proof of Theorem 2.

Proof of Theorem 2. Let $W=\left\{\left(x_{0}, x_{1}, \cdots, x_{m}\right): x_{0} \in V_{q}^{q}, x_{j}=A_{j} x_{0}\right.$, $j=1,2, \cdots, m\}$. Given a $q$-martingale $f$, as noted before, we have $T_{j} E f=A_{j} E f, j=1,2, \cdots, m$. The local differences of $E f, A_{1} E f, \cdots$, $A_{m} E f$ can be regarded as elements in $W$. Since $A_{j}$ 's do not have any common real eigenvector in $V_{q}^{q}$, the hypothesis of lemma is satisfied. Thus $\left|\left(E f, A_{1} E f, \cdots, A_{m} E f\right)\right|^{p}$ is a submartingale for some $p<1$.

By an usual martingale majorant argument, we have that $E f \in H^{1}$. Similarly, $G f \in H^{1}$. Therefore $f \in H^{1}$.

Another simple application of the lemma is by letting $m=1$ :

CoRollary 2. Let $W$ be a subspace of $V_{q}$ not containing any nonzero real vector. Then for a q-martingale $f$ having local differences in $W$, there exists a positive $p<1$ such that $|f|^{p}$ is a submartingale. Consequently, $\|f\|_{H^{1}} \leqq C\|f\|_{L^{1}}$ for some $C>0$ independent of $f$.

The fact that a $q$-martingale $f$ having local differences in such a $W$ can be regarded as an analytic property of $f$. The following is a version of the $F$. and M. Riesz theorem:

Corollary 3. Let $W$ be a subspace of $V_{q}$ not containing any 
nonzero real vector. If all local differences of the q-martingale corresponding to a finite measure $\mu$ are in $W$, then $\mu$ is absolutely continuous, moreover, $\mu^{*} \in L^{1}$.

4. Integrability and $H^{1}$. Our basic lemma in $\S 3$ enables us to characterize $H^{1}$ as the set of $f$ for which certain transforms are in $L^{1}$ without a priori assuming $f$ to be integrable. Special cases were considered before in [1].

Theorem 3. Suppose that $A_{j}, j=1,2, \cdots, m$, are linear operators from $V_{q}$ to $V_{q}$ such that there is no nonzero $x \in V_{q}$ for which all $A_{j} x$ 's are multiples of a common real vector in $V_{q}$. Let $T_{j}=T_{A_{j}}$ be the transform relative to $A_{j}, j=1,2, \cdots, m$. Then for $a$ $q$-martingale $f, f \in H^{1}$ if and only if $T_{j} f, j=1,2, \cdots, m$, are $L^{1}$ bounded.

Proof. Let $A$ be the direct product of $A_{1}, \cdots, A_{m}$, i.e., $A x=$ $\left(A_{1} x, \cdots, A_{m} x\right)$ for $x \in V_{q}$. Set $W=\left\{A x: x \in V_{q}\right\} \subset C^{m q}$. $W$ satisfies the condition in the lemma of $\S 3$ by the hypothesis. It follows from the lemma, by taking $a_{j}$ to be the corresponding values of $T_{j} f, j=1,2, \cdots, m$, that $\left|\left(T_{1} f, \cdots, T_{m} f\right)\right|^{p}=\left(\sum_{j=1}^{m}\left|T_{j} f\right|^{2}\right)^{p / 2}$ is a submartingale for some $p<1$. Thus by an usual argument, $\left(T_{j} f\right)^{*} \in L^{1}$, $j=1,2, \cdots, m$.

Now if $A x=0$ for some $x \in V_{q}$, then $A_{j} x=0$ for all $j$. From the hypothesis, we have $x=0$. Hence $A: V_{q} \rightarrow V_{q}^{m}$ is injective. Let $B$ be a left inverse of $A ; B A=I$. Namely, there exist $B_{j}: V_{q} \rightarrow V_{q}$, $j=1,2, \cdots, m$, such that $I=B A=\sum_{j=1}^{m} B_{j} A_{j}$. Let $S_{j}=T_{B_{j}}, j=$ $1,2, \cdots, m$, be the transform relative to $B_{j}$. As noted before such transforms preserve $H^{1}$, therefore we have $f=\sum_{j=1}^{m} S_{j} T_{j} f \in H^{1}$.

Since the converse is obvious, the proof is completed.

For the case that one of the operators is the identity, the result is obtained in [7].

5. Application to lacunary Fourier series. We shall apply the preceeding results to the martingales considered by Gundy and Varopoulos in [6].

Let $f \in L^{1}(\boldsymbol{T})$ with Fourier series expansion $\sum_{k} \hat{f}(k) \exp (i k \theta)$. Suppose $\hat{f}(0)=0$. Define $f_{n}(\theta)=q^{-n} \sum_{j=0}^{q^{n}-1} f\left(\theta+2 \pi j q^{-n}\right)=\sum_{k} \hat{f}\left(k q^{n}\right)$ $\exp \left(i k q^{n} \theta\right)$. Let $\mathscr{F}_{n}$ be the $\sigma$-field of all $2 \pi q^{-n}$-periodic Borel sets of $\boldsymbol{T}$. Then $\left\{f_{n}\right\}$ forms a backwards $q$-martingale relative to $\left\{\mathscr{F}_{n}\right\}$. The $H^{1}$ space of such martingales is denoted by $H_{q}^{1}$. The difference sequence of the martingale $f$ is given by

$$
d_{n}(\theta)=f_{n}(\theta)-f_{n+1}(\theta)=\sum_{k ; q \nmid k} \hat{f}\left(k q^{n}\right) \exp \left(i k q^{n} \theta\right) .
$$


For a fixed $\theta$ in an atom $S$ of $\mathscr{F}_{n+1}$, a collection of coset representatives of $S$ contained in an atom of $\mathscr{F}_{n}$ is $\left\{\theta_{j}\right\}_{j=0}^{q-1}$ where $\theta_{j}=\theta+$ $2 \pi j q^{-(n+1)}$. Hence if we let $x_{k}$ be the vector in $V_{q}$ with components $x_{k j}=\exp \left(2 \pi i j k q^{-1}\right), j=0,1, \cdots, q-1$, then the local difference $d_{n}$ of $f$ at $\theta$ in an atom of $\mathscr{F}_{n+1}$ is

$$
\sum_{l=1}^{q-1}\left[\sum_{s} \widehat{f}\left((s q+l) q^{n}\right) \exp \left(i(s q+l) q^{n} \theta\right)\right] x_{l} .
$$

Assume that $B \subset\{1,2, \cdots, q-1\}$ is such that $m \in B$ implies $q-m \notin B$ and let $W$ be the span of the vectors $\left\{x_{m}\right\}_{m \in B}$. There is no nonzero real vector in $W$ since if $y=\sum_{m \in B} a_{m} x_{m}$ is real in $W$, then $y=\bar{y}=\sum_{m \in B} \bar{a}_{m} \bar{x}_{m}=\sum_{m \in B} \bar{a}_{m} x_{q-m}$ which implies $y=0$. Let [k] denote the integer between 0 and $q-1$ such that $[k] \equiv k(\bmod q)$. We observe that $x_{k}=x_{[k]}$. Moreover, if $\hat{f}\left(k q^{n}\right)=0$ when $[k] \notin B$, then local differences of $f$ are in $W$. Therefore Corollary 2 gives the following

THEOREM 4. Let $B$ be any subset of $\{1,2, \cdots, q-1\}$ such that $m$ and $q-m$ are not both in $B$. Suppose

$$
\hat{f}\left(k q^{n}\right)=0 \text { for }[k] \notin B \text { and } n \geqq 0 \text {. }
$$

Then

$$
\|f\|_{H_{q}^{1}} \leqq C\|f\|_{L^{1}} \text { with some } C>0
$$

We show now how the result of Gundy and Varopoulos ([6]; Theorem 2 and Theorem 4) follows from this. For a subset $A$ of integers, we write $f_{A}(\theta)=\sum_{n \in A} \hat{f}(n) \exp (\operatorname{in} \theta)$. Assume that $q$ is odd. We choose, e.g.,

$$
B_{1}=\left\{1,2, \cdots, \frac{q-1}{2}\right\} \text { and } B_{2}=\left\{\frac{q+1}{2}, \cdots, q-1\right\}
$$

and set

$$
A_{i}=\left\{k q^{n}:[k] \in B_{i}, n \geqq 0\right\}, i=1,2 .
$$

Note that the nonzero integers $Z^{*}$ is a disjoint union of $A_{1}$ and $A_{2}$. Thus it follows from Theorem 4 that

Corollary 4. Suppose $q$ is odd and $A_{1}, A_{2}$ are given as above. Then $f \in H_{q}^{1}$ if and only if $f_{A_{1}}$ and $f_{A_{2}} \in L^{1}$.

(This is Theorem 2 of [6].)

When $q$ is even, the problem is more delicate as has been seen 
in [6]. We use the idea in $\S 2$ and consider the $q^{2}$-martingales $E f$ and $G f$ associated with the (backwards) $q$-martingale $\left\{f_{n}\right\}$ given above.

Recall that $E f$ is the $q^{2}$-martingale such that $E f=\sum d_{2 n}$ where $\left\{d_{n}\right\}$ is the martingale difference sequence of $\left\{f_{n}\right\}$. Also $d_{2 n}(\theta)=$ $\sum_{k ; q \nmid k} \hat{f}\left(k q^{2 n}\right) \exp \left(i k q^{2 n} \theta\right)$. Hence the local difference of $E f$ at $\theta$ is given by

$$
\sum_{1 \leqq<l q^{2}, q \nmid l} \sum_{s} \hat{f}\left(\left(s q^{2}+l\right) q^{2 n}\right) \exp \left(i\left(s q^{2}+l\right) q^{2 n} \theta\right) x
$$

where $x_{l}=\left\langle\exp \left(2 \pi i j l q^{-2}\right)\right\rangle_{j=0}^{q^{2}-1}$.

Now let, for instance

$$
B_{1}=\left\{l: 1 \leqq l<\frac{q^{2}}{2}, q \nmid l\right\} \quad \text { and } \quad B_{2}=\left\{l: \frac{q^{2}}{2}<l \leqq q^{2}-1, q \nmid l\right\} .
$$

Set $A_{i}=\left\{\left(s q^{2}+l\right) q^{2 n}: s \in Z, l \in B_{i}\right\}, i=1,2$. Note that $E f=f_{A_{1} \cup A_{2}}$ and $(E f)_{A_{i}}=f_{A_{i}}, i=1,2$. Therefore it follows from Theorem 4 that

$$
\left\|f_{A_{i}}\right\|_{H_{q^{2}}^{1}} \leqq C_{i}\left\|f_{A_{i}}\right\|_{L^{1}}, i=1,2 .
$$

The equivalence of the norms $\|\cdot\|_{H_{q}^{1}}$ and $\|\cdot\|_{H_{q^{2}}^{1}}$ gives

$$
\left\|f_{A_{i}}\right\|_{I_{q}^{1}} \leqq C_{i}\left\|f_{A_{i}}\right\|_{L^{1}}, i=1,2 .
$$

Similarly, let $A_{i+2}=\left\{\left(s q^{2}+l\right) q^{2 n+1}: s \in Z, l \in B_{i}\right\}, i=1$, 2 , (by considering $G f)$, we have

$$
\left\|f_{A_{i}}\right\|_{H_{q}^{1}} \leqq C_{i}\left\|f_{A_{i}}\right\|_{L^{1}}, i=3,4 \text {. }
$$

Since $A_{\imath}, i=1,2,3,4$, form a partition of the nonzero integers, we have

Corollary 5. Let $q$ be a positive even integer and $A_{i}, i=1,2,3,4$ be defined as above. Then $f \in H_{q}^{1}$ if and only if $f_{A_{i}} \in L^{1}, i=1,2,3,4$.

For $q=2^{k}$, this is Theorem 4(a) of Gundy-Varopoulos [6]. If $q$ is even but not a power of 2 , this improves their results by reducing the number of sets in the partition from six to four. Thus it provides an answer to the open problem posed by Gundy and Varopoulos [6], namely, at most three Fourier multipliers are needed to characterize $H_{q}^{1}$.

We remark here that the partition into $A_{i}, i=1,2,3,4$, is merely one of many choices in order that Corollary 5 is valid.

6. Application to local field analysis. We apply the main 
results to multiplier transforms on local fields. Preliminaries on Fourier analysis on local fields are found in [8].

Let $K$ be a local field with residue class field isomorphic to $G F(q) . \quad H^{1}(K)$ is the space of regular functions on $K \times \boldsymbol{Z}$ with integrable maximal functions. Let $m$ be a nonconstant bounded function on $K^{*}=K \backslash\{0\}$ such that for some integer $k \geqq 1, m(x+y)=$ $m(x)$ whenever $|y| \leqq q^{-k}|x|$. If $k$ is the smallest among such integers, we shall say that $m$ is ramified of degree $k$. If $m$ is homogeneous of degree 0 , then the mapping $f \rightarrow(m \hat{f})^{\vee}$ is a singular integral transform and a transform on $q$-martingales (i.e., regular functions on $K \times \boldsymbol{Z}$ ) of the type studied in connection with Theorem 2. (We note that previous results also hold for $\sigma$-finite measure spaces.) If $m$ is not homogeneous, then this mapping is of more general type which can be realized as applying a different operator $A^{(n)}$ on the atoms in each "level" $n$ (i.e., $\sigma$-field $\mathscr{F}_{n}$ ).

We begin with multipliers with ramification degree one:

THEOREM 5. Let $m_{j}, j=1,2, \cdots, n$, be bounded functions on $K$, ramified of degree 1 and such that $\sum_{j=1}^{n}\left|m_{j}(x)-m_{j}(-x)\right| \geqq c>0$, for $x \neq 0$. Then

$$
H^{1}(K)=\left\{f \in L^{1}: m_{j} \hat{f} \in \widehat{L^{1}}(K), j=1,2, \cdots, n\right\} .
$$

Proof. We regard a vector in $C^{q}$ as a function on the finite group (field) $G F(q)$. Let $W$ be the set of all vectors $\left(x_{0}, \cdots, x_{n}\right) \in$ $V_{q}^{n+1}$ satisfying $\hat{x}_{j}(g)=\alpha_{j}(g) \hat{x}_{0}(g), j=1,2, \cdots, n$, for some function $\alpha_{j}$ on $G F(q)$ with $\left|\alpha_{j}(g)\right| \leqq\left\|m_{j}\right\|_{\infty}$ and $\sum_{j=1}^{n}\left|\alpha_{j}(g)-\alpha_{j}(-g)\right| \geqq c>0$ for $g \neq 0$. $W$ satisfies the condition of the lemma in $\S 3$. For every sphere in $K$, the local differences of $f$ and $\left(m_{j} \hat{f}\right)^{2}, j=1,2, \cdots$, $n$, constitute an element in $W$. Thus, by the lemma,

$$
\left(|f|^{2}+\sum_{j}\left|\left(m_{j} \hat{f}\right)^{\vee}\right|^{2}\right)^{p / 2}
$$

is a submartingale (i.e., subregular) for some $p<1$. Therefore Theorem 5 follows as usual.

In the homogeneous case, this is proved in [7] and the condition is shown there to be necessary also.

A similar result without a priori assuming $f \in L^{1}$ is obtained by the method of Theorem 3. We state here one version for the homogeneous case:

Theorem 6. Suppose $m_{j}, j=1,2, \cdots, n$, are homogeneous of degree 0 and ramified of degree 1 . Then

$$
H^{1}(K)=\left\{f: m_{j} \hat{f} \in \widehat{L^{1}}(K), j=1,2, \cdots, n\right\}
$$


if and only if $\left\langle m_{j}(x)\right\rangle_{j=1}^{n}$ is not a constant multiple of $\left\langle m_{j}(-x)\right\rangle_{j=1}^{n}$ for any $x \neq 0$.

We now consider multipliers which are ramified of degree $k \geqq 2$ : Let, for $n \in Z$,

$$
e(x)=\left\{\begin{array}{l}
1 \text { if }|x|=q^{2 n} \\
0 \text { if }|x|=q^{2 n+1}
\end{array} \text { and } g(x)=1-e(x) .\right.
$$

If $E f$ and $G f$ are the $q^{2}$-martingales associated to a $q$-martingale (i.e., a regular function) $f$, then $\widehat{E f}=e \hat{f}$ and $\widehat{G f}=g \hat{f}$. Therefore we have

CoRollary 6. Suppose $m$ is homogeneous of degree 0 and ramified of degree 2 . Then

$$
\begin{aligned}
H^{1}(K) & =\left\{f: e \hat{f}, m e \hat{f}, g \hat{f}, m g \hat{f} \in \widehat{L^{1}}(K)\right\} \\
( & \left.=\left\{f \in L^{1}(K): m \hat{f}, e \widehat{f}, m e \widehat{f} \in \widehat{L^{1}}(K)\right\}\right),
\end{aligned}
$$

if and only if $m(x) \neq m(-x)$ for every nonzero $x \in K$.

Proof. We note that the linear operator $A$ on $V_{q}^{q}$ corresponding to the transform $f \rightarrow(m \hat{f})^{\vee}$ is a convolution on a finite group whose Fourier transform is the restriction of the multiplier $m$. If $m(x) \neq m(-x)$ for all nonzero $x \in K$, then $A$ does not have real eigenvectors (c.f. $[7 ; \S 4])$. Therefore the result follows from Theorem 2 and the discussion after it.

More generally, we let $e_{j}^{k}(x)=1$ if $|x|=k n+j$ and $e_{j}^{k}(x)=0$ otherwise, for $k \geqq 1, n \in Z, j=1,2, \cdots, k$. We have

CoROLlaRY 7. Suppose $m$ is homogeneous of degree 0 and ramified of degree $k$. Then

$$
H^{1}(K)=\left\{f: e_{j}^{k} \hat{f}, m e_{j}^{k} \hat{f} \in \widehat{L^{1}}(K), j=1,2, \cdots, k\right\}
$$

if and only if $m(x) \neq m(-x)$ for all $x \neq 0$.

We remark that various versions of the results similar to Corollary 6 and Corollary 7 that involve several multipliers $m_{j}$ 's being ramified of degree $k$ and/or nonhomogeneous also follow from Theorem 2 and Theorem 3. We omit the details here.

In characterizing $H^{1}(K)$, if $q$ is odd, it suffices to take an $m$ with ramification degree 1 (c.f. [1], [7]). If $q$ is even and thus a power of 2, then $K$ is an extension of a dyadic field or a dyadic series field. For the former case, a multiplier $m$ with ramification 
degree 2 will do. However, in an extension $K$ of a dyadic series field, $x=-x$ for all $x \in K$. Thus no finite set of multipliers of this type characterizes $H^{1}(K)$.

\section{REFERENCES}

1. J. -A. Chao, $H^{p}$ spaces of conjugate systems on local fields, Studia Math., 49 (1974), 267-287.

2. Conjugate characterizations of $H^{1}$ dyadic martingales, Math. Ann., 240 (1979), 63-67.

3. - The spaces $H^{p}$ and $B M O$ on regular martingales, preprint.

4. J.-A. Chao and M. H. Taibleson, A subregularity inequality of conjugate systems on local fields, Studia Math., 46 (1973), 249-257.

5. — Generalized conjugate systems on local fields, Studia Math., 64 (1979), 213-225.

6. R. F. Gundy and N. Th. Varopoulos, A martingale that occurs in harmonic analysis, Ark. Mat., 14 (1976), 179-187.

7. S. Janson, Characterizations of $H^{1}$ by singular integral transforms on martingales and $\boldsymbol{R}^{n}$, Math., Scand., 41 (1977), 140-152.

8. M. H. Taibleson, Fourier analysis on local fields, Princeton, 1975.

Received May 15, 1980.

Cleveland State University

Cleveland, OH 44115

AND

Institut MitTAG-LeffleR

AURAVAGEN 17

S-182 62 DJURSHOLM

SWEDEN 



\section{PACIFIC JOURNAL OF MATHEMATICS}

EDITORS

DONALD BABBITT (Managing Editor)

University of California

Los Angeles, CA 90024

Hugo RossI

University of Utah

Salt Lake City, UT 84112

C. C. MOORE and ANDREW OGG

University of California

Berkeley, CA 94720

\section{J. DugundjI}

Department of Mathematics

University of Southern California

Los Angeles, CA 90007

R. FINN and J. MILGRAM

Stanford University

Stanford, CA 94305

\section{ASSOCIATE EDITORS}
R. ARENS
E. F. BECKENBACH
B. H. NEUManN
F. WOLF
K. YoSHIDA

\section{SUPPORTING INSTITUTIONS}

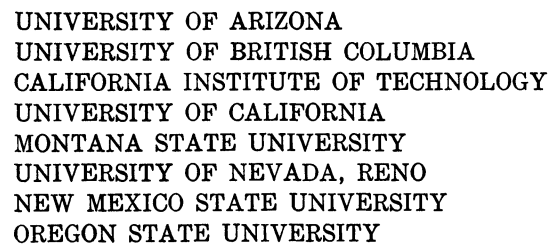

UNIVERSITY OF ARIZONA

UNIVERSITY OF BRITISH COLUMBIA

CALIFORNIA INSTITUTE OF TECHNOLOGY

UNIVERSITY OF CALIFORNIA

MONTANA STATE UNIVERSITY

UNIVERSITY OF NEVADA, RENO

NEW MEXICO STATE UNIVERSITY

OREGON STATE UNIVERSITY

\author{
UNIVERSITY OF OREGON \\ UNIVERSITY OF SOUTHERN CALIFORNIA \\ STANFORD UNIVERSITY \\ UNIVERSITY OF HAWAII \\ UNIVERSITY OF TOKYO \\ UNIVERSITY OF UTAH \\ WASHINGTON STATE UNIVERSITY \\ UNIVERSITY OF WASHINGTON
}

The Supporting Institutions listed above contribute to the cost of publication of this Journal, but they are not owners or publishers and have no responsibility for its content or policies.

Mathematical papers intended for publication in the Pacific Journal of Mathematics should be in typed form or offset-reproduced, (not dittoed), double spaced with large margins. Please do not use built up fractions in the text of the manuscript. However, you may use them in the displayed equations. Underline Greek letters in red, German in green, and script in blue. The first paragraph or two must be capable of being used separately as a synopsis of the entire paper. Please propose a heading for the odd numbered pages of less than 35 characters. Manuscripts, in triplicate, may be sent to any one of the editors. Please classify according to the scheme of Math. Reviews, Index to Vol. 39. Supply name and address of author to whom proofs should be sent. All other communications should be addressed to the managing editor, or Elaine Barth, University of California, Los Angeles, California, 90024.

50 reprints to each author are provided free for each article, only if page charges have been substantially paid. Additional copies may be obtained at cost in multiples of 50 .

The Pacific Journal of Mathematics is issued monthly as of January 1966. Regular subscription rate: $\$ 102.00$ a year (6 Vols., 12 issues). Special rate: $\$ 51.00$ a year to individual members of supporting institutions.

Subscriptions, orders for numbers issued in the last three calendar years, and changes of address shoud be sent to Pacific Journal of Mathematics, P.O. Box 969, Carmel Valley, CA 93924, U.S.A. Old back numbers obtainable from Kraus Periodicals Co., Route 100, Millwood, NY 10546.

\section{PUBLISHED BY PACIFIC JOURNAL OF MATHEMATICS, A NON-PROFIT CORPORATION}

Printed at Kokusai Bunken Insatsusha (International Academic Printing Co., Ltd.). 8-8, 3-chome, Takadanobaba, Shinjuku-ku, Tokyo 160, Japan. 


\section{Pacific Journal of Mathematics}

\section{Vol. 97, No. $2 \quad$ February, 1981}

Patrick Robert Ahern and N. V. Rao, A note on real orthogonal measures . . . . . 249

Kouhei Asano and Katsuyuki Yoshikawa, On polynomial invariants of fibered

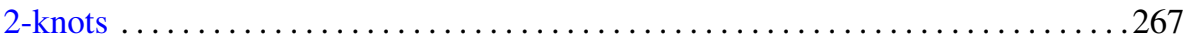

Charles A. Asmuth and Joe Repka, Tensor products for $S L_{2}(\mathscr{K})$. I.

Complementary series and the special representation

Gary Francis Birkenmeier, Baer rings and quasicontinuous rings have a

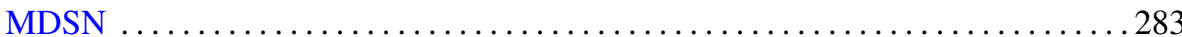

Hans-Heinrich Brungs and Günter Törner, Right chain rings and the generalized

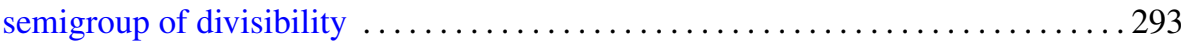

Jia-Arng Chao and Svante Janson, A note on $H^{1} q$-martingales . . . . . . . . . 307

Joseph Eugene Collison, An analogue of Kolmogorov's inequality for a class of

additive arithmetic functions

Frank Rimi DeMeyer, An action of the automorphism group of a commutative ring on its Brauer group

H. P. Dikshit and Anil Kumar, Determination of bounds similar to the Lebesgue

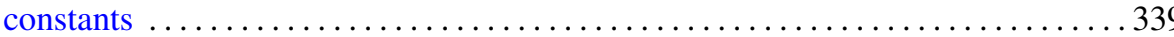

Eric Karel van Douwen, The number of subcontinua of the remainder of the plane

D. W. Dubois, Second note on Artin's solution of Hilbert's 17th problem. Order spaces

Daniel Evans Flath, A comparison of the automorphic representations of GL(3) and its twisted forms

Frederick Michael Goodman, Translation invariant closed $*$ derivations

Richard Grassl, Polynomials in denumerable indeterminates

K. F. Lai, Orders of finite algebraic groups

George Kempf, Torsion divisors on algebraic curves

Arun Kumar and D. P. Sahu, Absolute convergence fields of some triangular matrix methods

Elias Saab, On measurable projections in Banach spaces

Chao-Liang Shen, Automorphisms of dimension groups and the construction of AF algebras

Barry Simon, Pointwise domination of matrices and comparison of $\Phi_{p}$ norms

Chi-Lin Yen, A minimax inequality and its applications to variational inequalities

Stephen D. Cohen, Corrections to: "The Galois group of a polynomial with two indeterminate coefficients"

Phillip Schultz, Correction to: "The typeset and cotypeset of a rank 2 abelian group"

Pavel G. Todorov, Correction to: "New explicit formulas for the $n$th derivative of composite functions"

Douglas S. Bridges, Correction to: "On the isolation of zeroes of an analytic function" 